\title{
ECC
}

Eurasian Chemical Communications

\section{Preparation and study of starch/ collagen/ polycaprolactone nanofiber scaffolds for bone tissue engineering using electrospinning technique}

\section{Mohammad Ali Ghavimi ${ }^{a}$, Ramin Negahdaria, Amirhossein Bani Shahabadia, Simin Sharifib, Ezatolah Kazeminejadc, Shahriar Shahia,b,*, Solmaz Maleki Dizaj $^{\mathbf{b}, *}$}

a Faculty of Dentistry, Tabriz University of Medical Sciences, Tabriz, Iran

${ }^{b}$ Dental and Periodontal Research Center, Tabriz University of Medical Sciences, Tabriz, Iran

'Dental Research Center, Golestan University of Medical Sciences, Gorgan, Iran

Received: 13 October 2019, Accepted: 02 November 2019, Published: 23 November 2019

\begin{abstract}
Bone tissue engineering has been a fast growing area recently. It suggests a novel and talented method for bone regeneration. The aim of the current work is to produce nanofiber scaffolds based on starch/ collagen/ polycaprolactone (PCL) biomaterials by means of an electrospinning methodology for bone tissue engineering purposes. The results showed that the developed structures have good physicochemical and interconnected properties that could be considered for bone tissues engineering. The results from the characterization specified that the nanofibers were successfully prepared with monodispersed nanosized diameter $(60 \mathrm{~nm})$, uniform network shaped morphology and negative surface charge $(-13.5 \mathrm{mV})$. Besides, the applied method can be set up to prepare fiber-based structures using other polymeric materials. We believe that by incorporating different materials to reduce the degradation rate of the fibers, it can be matched with the speed of tissue regeneration. In this case, the prepared nanofibers can be used as the membrane biomaterials, for example, guided bone regeneration (GBR) membrane.
\end{abstract}

Keywords: Bone tissue engineering; nanofiber; electrospinning; guided bone regeneration.

\section{Introduction}

Bone tissue engineering is a fast evolving research area since it suggests a novel and hopeful method for bone regeneration purposes. Numerous necessities have been reported for engineering bone, such as selecting a cell type that matures/ differentiates into bone cells with the appropriate form and phenotype, regulating the growth factors and developing a ideal scaffold [1-3].

Electrospinning is an electrostaticbased one-step method to produce polymer/composite fibers or particles. The most basic component for this machine includes a reservoir (typically a

*Corresponding author: Shahriar Shahi, Solmaz Maleki Dizaj

Tel: +98 (041) 33353161, Fax: +98 (041) 33353161 Eurasian Chem. Commun., (2020) 122-127

E-mail: sshahriar32@gmail.com 
syringe), a pump, a high voltage power source and a collector. The electrospinning procedure initiates when the solution is pumped at a constant flow rate and a specific voltage is used to produce an electric field between the needle tip and the collector [4-7]. Recently, fibrous scaffolds in the field of tissue engineering have been one of the classes of synthetic biodegradable polymer-based carriers. Several applications of electrospun fibers have been testified to be the outcome of their specific features, mainly large surface area-to-volume ratio [7]. Starch-based structures have been reported for an extensive range of bone-related applications, including tissue engineering and drug delivery systems [8-11]. Natural origin, good mechanical possessions and high biocompatibility convert the starch-based resources to the beneficial materials in the biomedical area [12]. Besides, collagen, as the main constituent of connective tissue that shows excellent biocompatibility, once was used in tissue engineering. Type I and III collagens derived from porcine, bovine, and human are mostly used in the production of guided bone regeneration (GBR) membrane [13,14].

Nanofibrous membranes have attracted great attention due to high porosity and particular surface area and the high similarity to extracellular matrix (ECM). In the large scale, GBR membranes with nanofiber structures are more suitable for cell growth than traditional scaffolds. Thus, there is an a critical need to prepare more bioactive nanofibrous GBR membranes [15-19].

Polycaprolactone (PCL) is a linear aliphatic polyester. It is a hydrophobic, biocompatible, and moderately slowdegrading polymer, with broadly used biomedical applications. It is a thermoplastic polymer with proper stability and simple processability [20].
In the current study, we have established a new fibrous scaffold based on starch for bone tissue regeneration

\section{Experimental}

\section{Materials}

All the reagents used (starch, collagen ((derived from bovine)), polycaprolactone, chloroform, and dimethylformamide) were of analytical grade and prepared from Sigma-Aldrich, Germany.

\section{Nanofiber preparation method}

A mixture of starch/ collagen/ polycaprolactone $(50 / 15 / 35 \mathrm{wt} \%)$ were prepared and then by dissolving $0.5 \mathrm{~g}$ of this blending in $5 \mathrm{~mL}$ chloroform and 2.5 $\mathrm{mL}$ of dimethylformamide (DMF), the polymer solution was obtained. The obtained polymer solution was then electrospun using an electrospinning machine (ANSTCo RN/I, Iran). In this process, the prepared polymer solution was located in a capillary tube in a vertical form. A four-sided collector was applied to collect the nanofibers over the electrospinning jets (13 kV voltage, distance of $15 \mathrm{~cm}$ between a collector and a capillary tube for $15 \mathrm{~s}$ and $0.8 \mathrm{ml} / \mathrm{h}$ flow rate).

\section{Scaffold characterization}

\section{The mean fiber diameter}

The mean fiber diameter of the prepared nanofibers was measured by Dynamic Light Scattering (DLS) method (Malvern, United Kingdom) at $25{ }^{\circ} \mathrm{C}$.

Scanning electron microscopy (SEM) The morphology of the ready nanofibers was detected by a Scanning Electron Microscope (SEM, TESCAN, Warrendale, PA). The nanofibers were fixed via an adhesive tape on aluminium stubs and covered with gold before the examination.

\section{Zeta potential measurements}

Zeta potential measurements were done using zeta-sizer (Malvern, UK) at $25^{\circ} \mathrm{C}$. 
The freshly prepared suspension of nanofibers was diluted with distilled water and inserted into the capillary cell of the zeta-sizer machine.

\section{Tomography measurements}

Micro-computerized tomography (Scanco Medicals, Switzerland) with Xrays of $40 \mathrm{keV}$ and high-resolution was used to prepare tomography images of the prepared nanofibers.

\section{Mechanical properties}

The mechanical possessions of the nanofibers were tested by means of an INSTRON 4301 (Instron Int. Ltd., High Wycombe, UK).

\section{Results and discussion}

The mean diameter of nanofibers is a main and key parameter that can influence on different properties of nanofiber in biomedical applications $[7,19$, and 20]. The mean diameter of the produced starch-based nanofibers was measured to be about $60 \mathrm{~nm}$ with polydispersity index (PDI) value of 0.23 that shows the relatively monodispersed nanofibers (Figure 1). Other investigators have reported similar results for the nanofibrous scaffolds [21]. The goal of electrospinning procedure optimization is to discover the conditions of producing fibers with minimum diameter. Optimum conditions in our work were: $50 / 15 / 35 \mathrm{wt} \%$ of starch/ collagen/ polycaprolactone, $13 \mathrm{kV}$ voltage, distance of $15 \mathrm{~cm}$ between a collector, a capillary tube for $15 \mathrm{~s}$ and 0.8 $\mathrm{mL} / \mathrm{h}$ flow rate. The prepared scaffolds present a nanobridge shaped morphology as it can be seen in Figure 2. It can also be observed from the SEM image that the nanofibers were randomly electrospun on the collector with high similarity to the construction of the ECM. Other studies have reported comparable results for the nanofibrous scaffolds [21-22]. Furthermore, the nanofibers showed a network structure without the presence of beads. The diameters achieved by the DLS for the prepared nanofibers were different from the size obtained by the SEM images. It might be happened owing to an error in the Stokes-Einstein equation for DLS method (for viscosity magnitudes) [23].

The determined average of zeta potential of the nanofibers was $-13.5 \pm$ $0.17 \mathrm{mV}$ (Figure 3), indicating fiber stability being the repulsive charge between the fibers enough to avoid agglomeration [24-25]. It has also been reported that the negative zeta potential shows a significant promising impact on the attachment and proliferation of the bone cells. The reports showed that a surface with negative charge is more accessible for the attachment and proliferation of osteoblasts than neutral or positive charges [5 and 26]. The authors believed that these results established that the processing parameters were effectively optimized for producing fine nanofibers based on previous reports.

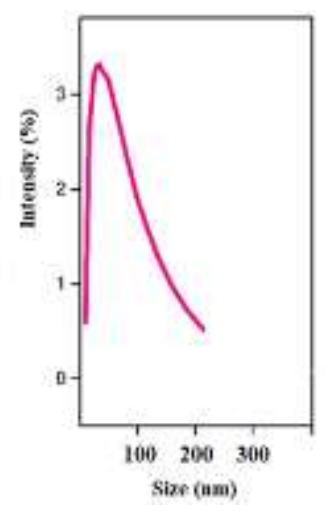

Figure 1. The mean fiber diameter of the prepared nanofibers. 


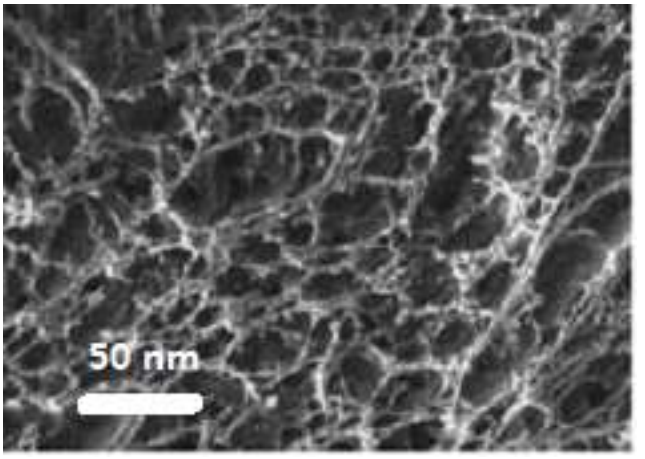

Figure 2. SEM images of the prepared nanofibers.

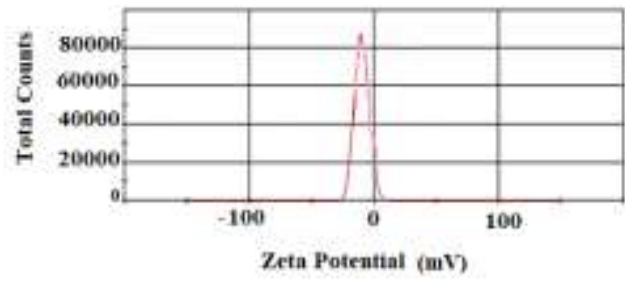

Figure 3. Zeta potential of the prepared structures-

Tomography using a micro-computed device lets better imagining of the inner structure of the prepared nanostructures [28]. It can help to define the relationship between structure, defects and critical failure and can be proper realistic imaging [28]. Figure 4 reveals that the macroporous structure of the nanofibrous structure is regular and homogeneous throughout all the volume of the sample and has good interconnectivity.

The porosity and interconnectivity are complex parameters that are currently acknowledged in the tissue engineering literature. According to previous reports, cell survivability depends on the pore networks that have to be optimized for molecular transport which is a function of interconnectivity. The organization of the pore walls is an another factor that should be studied. This measurement can be performed using gas adsorption. However, the influence of an anisotropic arrangement of pore struts on cell behaviour has not yet been sufficiently studied [27]. There is no verified standard for interconnectivity measurement. Tomography images also present the porosity and interconnectivity information about the prepared samples. Qualitative observation of Figure 4 shows high porosity and interconnectivity between pores that are two proper characteristics for a bone related structure. Wang et al. reported the use of nanofibers as a new scaffold for ultrafiltration purposes. Their tomography images showed a three-tier composite structure for the prepared electrospun nanofibrous. They stated that the electrospun nanofibrs can provide a well interconnected porous network with a large specific surface area for different biomedical application [26].

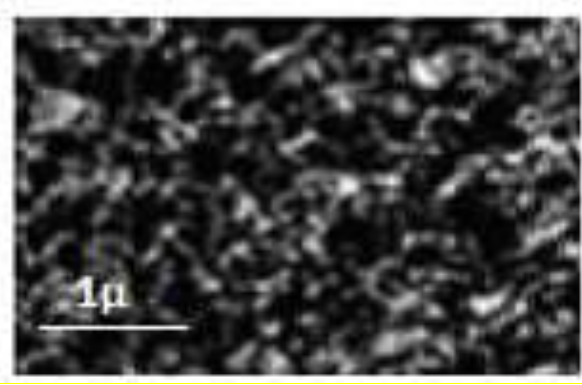

Figure 4. 2D model of representative micro-CT images of the prepared nanofibers

Mechanical properties of the prepared fibers were tested using compression tests. Figure 5 shows stress deformation of the nanofibers. There are so little details about stress-strain curves or deformation mechanisms of nanofibers in the published scientific literature. The stress-strain manners of the perepared nanofibrous structure is determined by an initial linear region. It seems that a plastic yielding, followed by strainhardening at constant stress-strain slope, is observed in this curve. Plastic deformation is owing to inter-fiber slippage rather than plastic deformation in the nanofibrous structure themselves 
[29]. Some investigators are believed that strain-hardening is desirable for nanofibers for biomedical applications as it comes to resist an unexpected excess in a direction of human muscles [30-33].

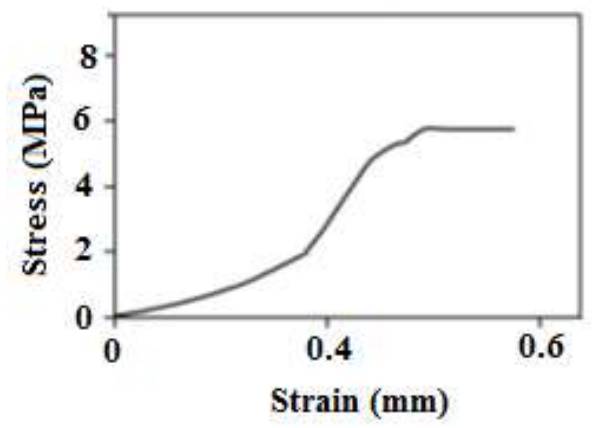

Figure 5. Stress deformation curve of the prepared nanofibers at $45^{\circ} \mathrm{C}$ and 100 bar.

\section{Conclusion}

The purpose of the present study was to yield nanofiber scaffolds from starchbased materials by means of an electrospinning method for bone tissue engineering purposes. Our outcomes The results showed that the prepared structures are supposed to show great potential for bone tissues. We believed that cross-linking methods can improve the mechanical possessions of the prepared fibrous structures. Besides, by incorporating different materials to reduce the degradation rate of the fibers, it can be matched with the speed of tissue regeneration. In this case, the prepared nanofibers can be used as the membrane biomaterials, for example GBR membranes. The prepared nanofibers with good physicochemical and interconnected properties of a starchbased material using a simple technology starts a novel methodology for tissue engineering. We believed that this method could be set up to prepare fiberbased structures using other polymeric materials. In our future studies, we will focus on the cross-linking of these nanofibers and GBR membrane designing from them.

\section{Acknowledgments}

This article was written based on a dataset from a research thesis registered at Tabriz University of Medical Sciences, Dental and Periodontal Research Center (number 63005). The thesis was supported by the Vice Chancellor for Research at Tabriz University of Medical Sciences that is greatly acknowledged.

\section{References}

[1] S. Maleki Dizaj, M. Barzegar-Jalali, M.H. Zarrintan, K. Adibkia, F. Lotfipour, Pharm. Sci., 2015, 20, 175182.

[2] L. Kumar Meena, H. Rather, D. Kedaria, R. Vasita, Int. J. Pol. Mater. Pol. Bio. 2019, 1, 1-17.

[3] A. Reddi, K. Iwasa. Principle. Reg. Med., 2019, 1, 405-416.

[4] K. Adibkia, S. Yaqoubi, S. Maleki Dizaj, Pharmaceutical and Medical Applications of Nanofibers. Novel Approaches for Drug Delivery, IGI Global, 2017, 338-63.

[5] A. Jahangiri, M. Barzegar-Jalali, Y. Javadzadeh, H. Hamishehkar, K. Adibkia. Artif. Cell. Nanomed. Biotechnol., 2017, 45, 1138-1145.

[6] L. Barghi, A. Jahangiri, J. Adv. Chem. Pharm. Mater., 2018, 1, 26-28.

[7] A. Jahangiri, K. Adibkia, BioImpacts., 2016, 6, 1-2.

[8] A.R.C. Duarte, J. F. Mano, R. L. Reis, J. Supercrit. Fluid., 2009, 49, 279-285.

[9] Z. Hadisi, J. Nourmohammadi, J. Mohammadi, Ceramic. Inter., 2015, 41, 10745-10754.

[10] A. Ghaee, S. Bagheri-Khoulenjani, H.A. Afshar, H. Bogheiri, Compos. Part B. Eng., 2019, 1, 107339-107341.

[11] F. Mirab, M. Eslamian, R. Bagheri, Biomed. Phys. Eng. Exp., 2018, 4, 055021-0055022.

[12] F.R. Maia, V.M. Correlo, J.M. Oliveira, R.L. Reis, Principles of 
Regenerative Medicine, Elsevier, 2019, 535-558.

[13] N. Alves, I. Leonor, H.S. Azevedo, R. Reis, J. Mano, J. Mate. Chem., 2010, 20, 2911-2921.

[14] K. Lin K, D. Zhang, M.H. Macedo, W. Cui, B. Sarmento, G. Shen, $A d v$. Function. Mater., 2019, 29, 18049431804944.

[15] M.C. Bottino, V. Thomas, G. Schmidt, Y.K. Vohra, T. M. G. Chu, M. J. Kowolik, Dent. Mater., 2012, 28, 703721.

[16] X. Song X, F. Ling, L. Ma, C. Yang, X. Chen X, Compos. Sci. Tech., 2013, 79, 8-14.

[17] J. Xue J, M. He, Y. Niu, H. Liu, A. Crawford, P. Coates P, Int. J. Pharm., 2014, 475, 566-577.

[18] F. Yang, S.K. Both, X. Yang, X.F. Walboomers, J.A. Jansen, Acta. Biomater., 2009, 5, 3295-3304.

[19] M. Tallawi, D. Dippold, R. Rai, D. D'Atri, J. Roether, D. Schubert, Mater. Sci. Eng C., 2016, 69, 569-576.

[20] C. Chu, Y. Wang, Y. Wang, R. Yang, L. Liu, S. Rung, Mater. Sci. Eng. C., 2019, 99, 73-82.

[21] N. Gómez-Cerezo, L. Casarrubios, M. Saiz-Pardo, L. Ortega, D. Pablo, I. Díaz-Güemes, Acta. Biomater., 2019, 1, 40-41.

[22] S. Maleki Dizaj, F. Lotfipour, M. Barzegar-Jalali, M.H. Zarrintan, K. Adibkia, Artif. Cell. Nanomed. Biotechnol., 2016, 44, 1475-1481.
[23] B.J. Berne, R. Pecora, Courier Corporation, 2000, 1-9.

[24] S. Maleki Dizaj, F. Lotfipour, M. Barzegar-Jalali M, M.H. Zarrintan, K. Adibkia, Artif. Cell. Nanomed. Biotechnol., 2017, 45, 535-543.

[25] S. Mirzaeei, K. Berenjian, R. Khazaei, Adv. Pharm. Bulletin., 2018, 8, 21-28.

[26] X. Wang, X. Chen, K. Yoon, Environ. Sci. Technol., 2005, 39, 76847691.

[27] S. Wang, F. Hu, J. Li, S. Zhang, M. Shen, M. Huang M, Nanomed, Nanotechnol. Biol. Med., 2018, 14, 2505-2520.

[28] J. Song, G. Zhu, H. Gao, L. Wang, N. Li, X. Shi, Bio. Design. Manufactur., 2018, 1, 254-264.

[29] S. Maleki Dizaj, F. Lotfipour, M. Barzegar-Jalali, M.H. Zarrintan, K. Adibkia, J. Drug. Deliv. Sci. Technol., 2016, 35, 16-23.

[30] G.L. Re, F. Lopresti, G. Petrucci, R. Scaffaro, Micron., 2015, 76, 37-45.

[31] S.T. Ho, D.W. Hutmacher, Biomater., 2006, 27, 1362-1376.

[32] J. Sheng, Y. Li, X. Wang, Y. Si, J. Yu, B. Ding, Sep. Purif. Technol., 2016, 158, 53-61.

[33] P.V. Kolluru, J. Lipner, W. Liu, Y. Xia, S. Thomopoulos, G.M. Genin, Acta. Biomate., 2013, 9, 9442-9450.

How to cite this manuscript: Mohammad Ali Ghavimi, Ramin Negahdari, Amirhossein Bani Shahabadi, Simin Sharifi, Ezatolah Kazeminejad, Shahriar Shahia, Solmaz Maleki Dizaj. Preparation and study of starch/ collagen/ polycaprolactone nanofiber scaffolds for bone tissue engineering using electrospinning technique . Eurasian Chemical Communications, 2020, 2(1), 122-127. 